\title{
HYPERTENSION INTERVENTION AT THE BARBERSHOP
}

According to a new study presented at the ACC Scientific Sessions 2018 in Orlando, FL, USA, health promotion in barbershops is an efficient way to reduce blood pressure (BP) in African-American men, a difficult-to-reach population with a high rate of uncontrolled hypertension.

Barbershops are potential sites for community health promotion, but the effectiveness of a barbershop-based programme to reduce BP levels in African-American men with hypertension has never been evaluated. This study included 319 African-American men with systolic BP $\geq 140 \mathrm{mmHg}$ : 132 clients from 28 barbershops were assigned to a pharmacist-led intervention in which the barbers encouraged meetings with a specialty-trained pharmacist inside the shop and 171 clients from 24 barbershops received a control approach in which barbers only promoted to follow up with a primary care provider and lifestyle changes. In the intervention group, the pharmacist regularly checked BP levels, prescribed antihypertensive medication, and sent notes to the participants' doctors. After 6 months, the reduction of mean systolic BP was $21.6 \mathrm{mmHg}$ greater in the intervention group compared with the control group, and more participants achieved the pre-specified BP goal of $\leq 130 / 80 \mathrm{mmHg}$ in the intervention group than in the control group $(63.6 \%$ versus $11.7 \%)$. The intervention increased the use of antihypertensive medication: after 6 months, $100 \%$ of participants in the intervention group used antihypertensive therapy compared with $63 \%$ in the control group.

Several factors can explain the efficacy of the intervention: easy access to the drugs and regular visits to the barbershops are a favourable setting to monitor and manage chronic diseases such as hypertension. Extending this community health promotion programme to more barbershops or to other social meeting points could help to treat approximately 3.5 million African-American men with $B P \geq 130 / 80 \mathrm{mmHg}$, who are now considered to have hypertension according to the new 2017 American BP guidelines. An ongoing study will inform on the sustainability beyond 6 months in BP reductions achieved with this approach.

Alexandra Le Bras 